\title{
Implementation and Application of PED Receiver Acquisition
}

\author{
Shanshan Cai ${ }^{1, a}$, Jiayi Chen ${ }^{1, b}$, Wei Yang ${ }^{1, c}$, Shu Diao ${ }^{1, d}$ \\ ${ }^{1}$ College of Instrumentation\& Electrical Engineering, Jilin University, Changchun 130026, China \\ a292071586@qq.com, ${ }^{\mathrm{b}} 1838029136 @ q q . c o m,{ }^{\mathrm{c}} 131684690 @ q q . c o m,{ }^{d}$ diaoshu@jlu.edu.cn
}

Keywords: PED; Phase sensitive detection; coding

\begin{abstract}
In view of the adverse effects brought by the noise in mine on the ultra-low signal detection, this article puts forward a hardware circuit design of the receiving system and a phase-sensitive detection algorithm which can improve SNR greatly and optimize the system. In addition, an original coding program for synchronization is made to apply to the wireless emergency communications system-PED(Personal Emergency Device ${ }^{[12]}$. Through the simulation experiment on PED, it can be concluded that the system is of high detection capability and sensitivity up to $0.75 \mathrm{~V} / \mathrm{nT}$ and $50 \mathrm{~Hz}$ frequency interference attenuation decreases by $-45 \mathrm{db}$. Furthermore, the receiver has strong ability to suppress noise so that faint signal can be detected from where the signal to noise ratio (SNR) is -58db and can decode the information from inoue to mine efficiently
\end{abstract}

\section{Introduction}

Nowadays, the wireless emergency communication system which was represented by PED has broad prospects $^{[1]}$, especially for mine calamity. For instance it can ensure stable communication while other systems are damaged and the rescue stuffs can take emergency measures in time so as to minimize casualties. Meanwhile, the PED system can communicate with any miner below which make production scheduling efficient and personnel deployment convenient, thus making the production efficiency greatly improved. However, the underground environment is complex, the existence of frequency harmonic noise, presence of random noise and noise spikes make it difficult to receive and process signals in PED system ${ }^{[4]}$. In consequence, taking appropriate technique and methods for detecting weak signal in complex noise environmental is the key to data processing. Since the phase-sensitive detector can identify the phase of the modulation signal and has the ability to select frequency, therefore basing on resonance reception in front of the receiver it can further improve the $\mathrm{Q}$ value and reduce the bandwidth so that the fixed frequency desired signals can be obtained among measuring signals. This article presents an idea to combine the hardware filtering with digital phase-sensitive detection and apply the method to data processing on weak signal to PED receiving system, which not only simplifies the circuit design but also makes the receiving device compact and portable. In addition, the SNR(Signal to Noise Ratio) can be improved significantly among complex environment. The speed of coding is fast and the ability to coding identification is first-class, all of which contribute to optimizing the various information transfer function from inoue to mine.

\section{Hardware design}

In order to filter out noise in the signal, we combine analog filter with a digital filter. The design ideas are as Fig.1:

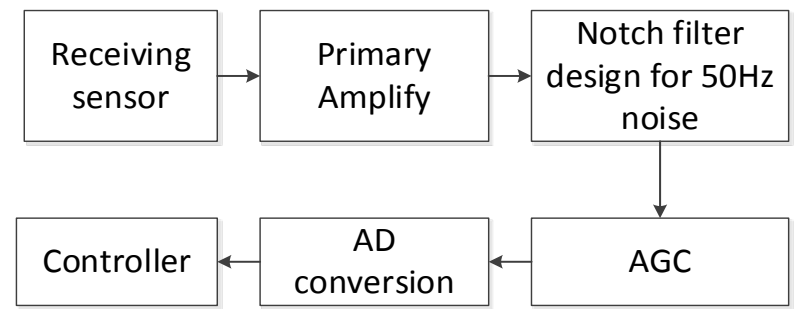

Fig.1 PED hardware design 
Because of the attenuation through the earth, the signal reaching the mine is already very weak and SNR is very low. In order to improve the detection capability of the system, high-sensitivity magnetic inductive sensors are put into use. In order to maximum the ability of detection, we take the advantage of the resonance and magnetic feedback, resulting in the detection accuracy up to $0.75 \mathrm{~V} / \mathrm{nT}$. Schematic diagram are shown in Fig 2.

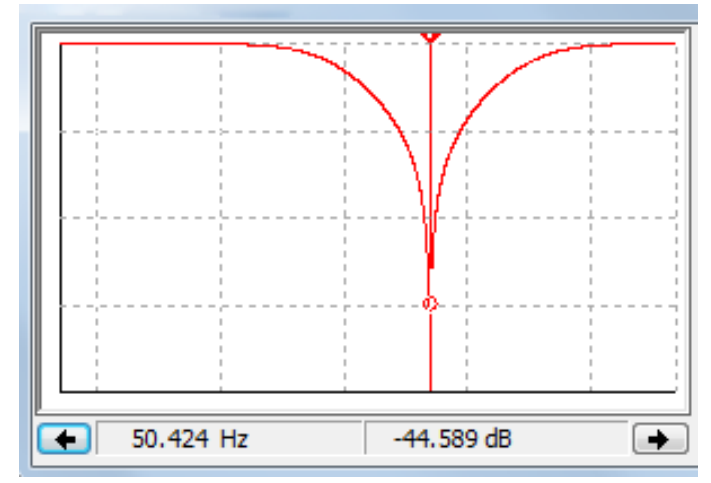

Fig .2 schematic inductive magnetic sensors

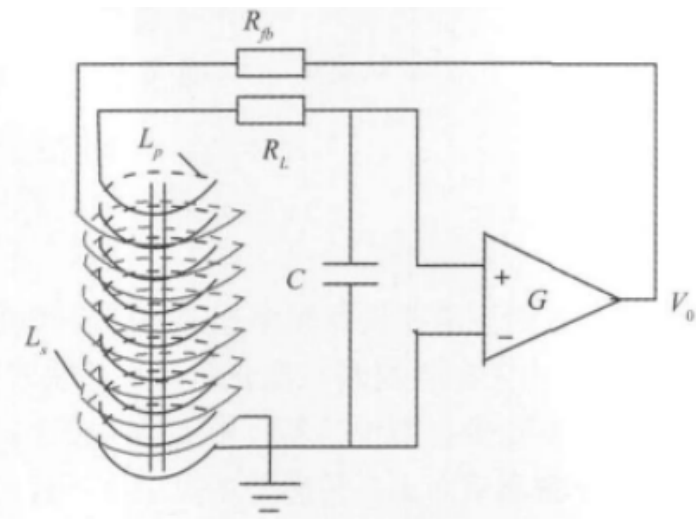

Fig .3 Bode plot for $50 \mathrm{~Hz}$ notch filter

In order to reduce the noise introduced into the system as much as possible, Ultra-low noise chip named AD797 was chosen for the Primary amplifier ${ }^{[2]}$. AD797 is the audio amplifier with very low noise of $0.9 \mathrm{nV} \sqrt{\mathrm{Hz}} @ 1 \mathrm{KHz}$ and the low distortion of $-120 \mathrm{db} @ 20 \mathrm{KHz}$ and the low input offset voltage of $80 \mathrm{uV}^{[3]}$, which was qualified for the primary amplifier.

In the underground environment, $50 \mathrm{~Hz}$ noise is the main noise signal. In order to improve the signal to noise ratio, notch circuit was purposely designed for the Interference of $50 \mathrm{H}$.The methods of Wien Bridge, double-T circuit, inverting band pass circuit and inductor simulation are the traditional way to design the notch. In this article, the last method, namely the inductor simulation method was put into use. Circuit is shown in Figure 4. Through the experiments, we found that signal of $50 \mathrm{~Hz}$ attenuation reached $45 \mathrm{db}$ through the notch.

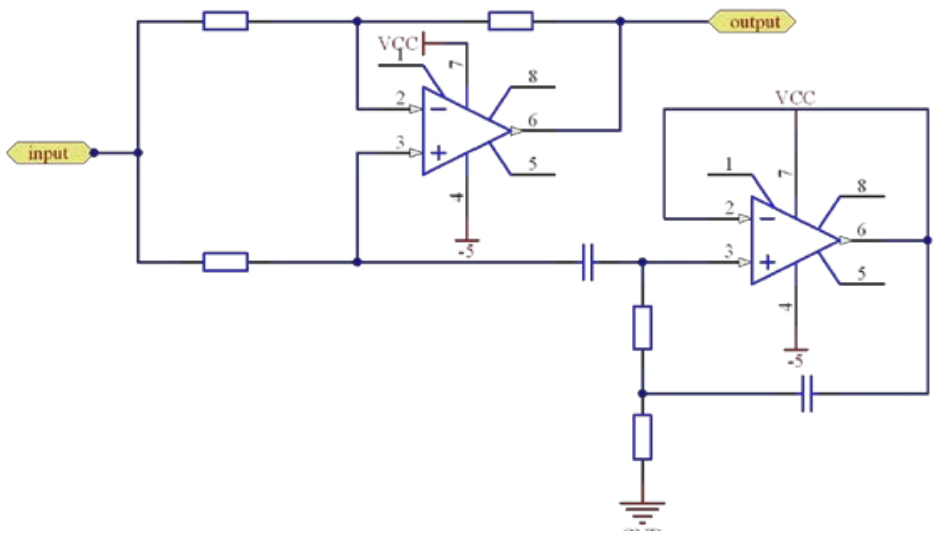

Fig.4 Design for $50 \mathrm{~Hz}$ notch filter

In order to combine digital phase sensitive detector with the analog phase sensitive detector, signal was applied by a DC bias before the AD converter, thereby the defect that negative voltage was not available for the AD converter. Taking into account the different locations of the received signal amplitude is different. In order to reduce the difficulty of programming, we amplified signal voltage to the half around of the reference voltage. Thereby, programmable amplifier chip AD603 was chosen for the automatic gain control circuit, namely AGC. Finally, signal amplitude is controlled at about $1.25 \mathrm{~V}$.

\section{Phase sensitive detection principle}

Although it's really difficult to make an analog filter with high Q-value, the phase-sensitive detector which has the same function as high Q-value narrowband band-pass filter can make up these shortcomings. Specifically, the Q-value of the phase-sensitive detector is up to $10^{8}$ thus only 
the signal which has the same frequency as the reference signal can pass making the frequency selection effect excellent ${ }^{[5][6]}$. On the other hand, the stability of the phase-sensitive detector frequency can reach $10^{-8}$, which ensures that the center frequency does not drift and stay stable. Ideally, the phase-sensitive detection filter bandwidth can be infinitely narrow so that detector has the function of single-frequency detection. If it is applied to receiving part of PED, the signal-noise ratio on useful signal can be greatly improved. Principle of analog phase-sensitive detector is shown in Fig 5.

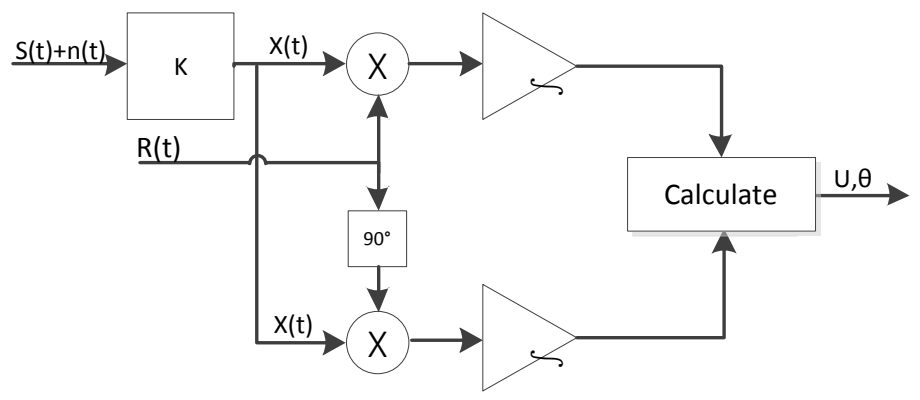

Fig.5 Schematic diagram of analog circuit for phase sensitive detection

Specifically, $\mathrm{S}(\mathrm{t})$ is the name of desired signal to be extracted and $\mathrm{R} 1(\mathrm{t})$ 、 $\mathrm{R} 2(\mathrm{t})$ are orthonormal reference signal, furthermore, $\mathrm{n}(\mathrm{t}), \mathrm{K}$ and $\mathrm{X}(\mathrm{t})$ stand for noise ,preamp gain and pre-amplified signal respectively. The main noise doped in the receiving ultra-low frequency signal through rock are $50 \mathrm{~Hz}$ frequency interference, $2-10 \mathrm{~Hz}$ geomagnetic signal interference, a little harmonics of Standard emission signal and some Gaussian noise, thermal noise from devices. Each signal can be represented as below

$$
\begin{gathered}
\mathrm{S}(\mathrm{t})=\mathrm{U}_{0} \sin \left(\omega_{0} t+\theta\right) \\
\mathrm{n}(\mathrm{t})=\mathrm{U}_{r 1} \cos \left(2 \pi \times 50 \times t+\theta_{r 1}\right)+\mathrm{U}_{r 2} \cos \left(\omega_{r 2} t+\theta_{r 2}\right)+\mathrm{N}(\mathrm{t}) \\
\mathrm{R}_{1}(\mathrm{t})=\sin \left(\omega_{0} \mathrm{t}\right) \\
\mathrm{R}_{2}(t)=\cos \left(\omega_{0} t\right)
\end{gathered}
$$

From the coherence algorithms on Figure1 the formulas can be concluded that

$$
\begin{gathered}
\mathrm{U}_{1}=\frac{1}{T} \int_{0}^{T} X(t) R_{1}(t) d(t)=\frac{K U_{0}}{2} \cos \theta \\
\mathrm{U}_{2}=\frac{1}{T} \int_{0}^{T} X(t) R_{2}(t) d(t)=\frac{K U_{0}}{2} \sin \theta
\end{gathered}
$$

The amplitude and phase of the analog phase sensitive detector can be calculated as

$$
\begin{aligned}
& \mathrm{U}=2 \times \sqrt{\mathrm{U}_{1}{ }^{2}+\mathrm{U}_{2}{ }^{2}}=K U_{0} \\
& \theta=\tan ^{-1}\left(U_{2} / U_{1}\right)
\end{aligned}
$$

However, there are inevitable shortcomings in analog phase-sensitive detector ${ }^{[10][13]}$. For instance, it has demanding requirement on accuracy of the device and the stability of external standard quadrature reference signals. Many complicated factors like harsh environment, temperature and humidity can cause drift of center frequency in phase sensitive detector and bring thermal noise easily. In addition, there will be unpredictable deviation in the useful signal calculation of amplitude and phase for the reason that DC bias amount brought by pre-hardware circuit is difficult to remove. In view of this, the method of digital phase-sensitive detection is taken to simplify the structure of the receiving device and improve accuracy on weak signal detection. The bandwidth of digital phase-sensitive detection is related to its sampling frequency and the number of sampling points ${ }^{[1]}$

$$
\mathrm{K}(\Delta \omega)=\frac{1}{N} \cdot\left|\frac{\sin \left(N \Delta \omega / 2 f_{c}\right)}{\sin \left(\Delta \omega / 2 f_{c}\right)}\right|
$$

Among the formula, fc, $\mathrm{N}, \Delta \omega$ and $\mathrm{K}$ stand for reference frequency, the number of sampling points, the gain, and the angular frequency difference between signal and standard reference angular frequency respectively. The conclusion from the formula is that the amplitude-frequency characteristics of $\operatorname{DSPD}^{[7][8]}$ algorithm are not only related to the frequency of the input signal but also the number of sampling points. The more points we sample, the better filtering effect it will have on out-band noise. Meanwhile, Figure 6 shows that as $\mathrm{N}$ increases the passband will be narrowed and the system will have better selective characteristics, thus it will be beneficial to filtering out noise. 


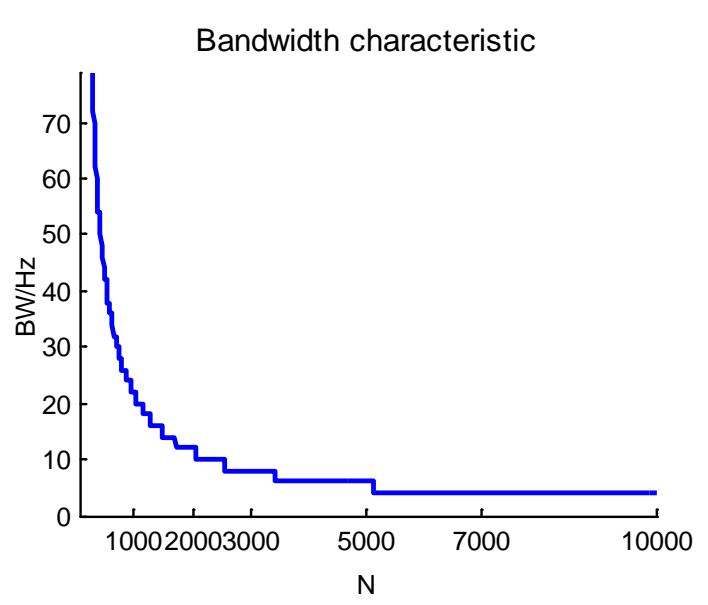

Fig.6 Diagram of the relation between sampling number and bandwidth

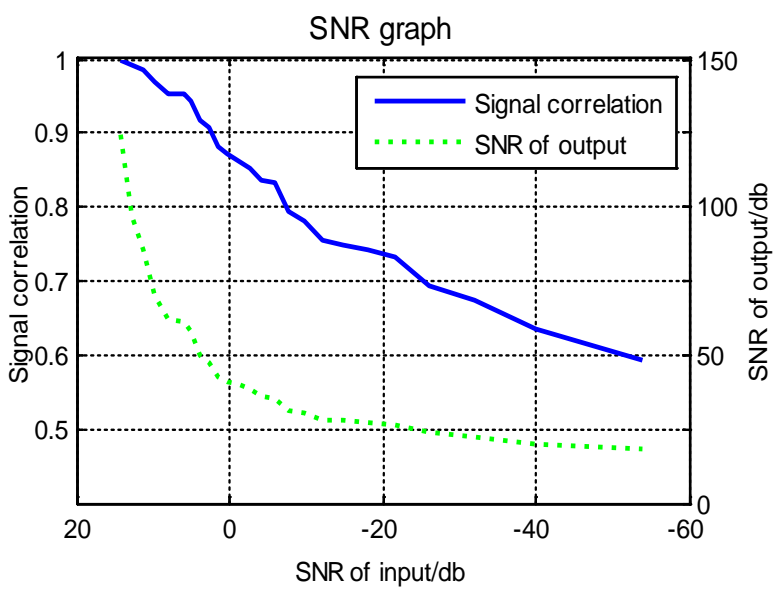

Fig.7 graph of SNR

After testing the detection capability of digital phase sensitive detector by inputting signals with different SNR, Figure 7 can be depicted as the SNR graph. The conclusion drew out from the graph is that digital phase sensitive detector can improve the Signal to noise ratio to a large extent. Supposing that the standard of receiving signal in PED receiving system is judged by signal dependency over 0.6 in the environment, the least SNR received by the system is $-58 \mathrm{db}$.

\section{Coding design and implementation}

PED system, which can achieve well down-hole information transfer and play a significant role in the promoting production rate and disaster prevention, is inseparable from reasonable and efficient coding techniques. Because the required information size and the transfer rate is relatively unimportant, the code communication method can meet the requirements of the commonly information transmission. Therefore, the code design adopt the binary code and based on $2 \mathrm{ASK}^{[4]}$ modulation we creatively propose the encoding method which match the anterior signal conditioning modules.

The signal correlation module DPSD confirms the transmitted center frequency through detecting the received signal's amplitude and phase information. To ensure stable and accurate DPSD results, the algorithm separates collection and calculation process. However this will lead to blind zone to the certain signal receiver which jeopardizes the decoding identification. After taking DPSD communication rate and bandwidth characteristics into consideration, a 25 times of transmitted center signal of the period of time is regarded as a unit time length to determine the bit signal (0 or 1$)$.

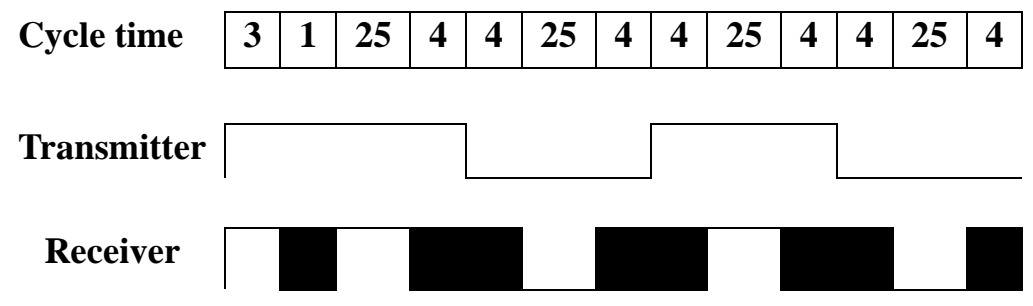

Fig.8 encoding timing diagram

The receiver receives the complete signal information in 25 transmitted cycles' time as pickup window, and takes 8 cycles' time for information processing computations. The mono-polar waveform information receiver defines 25 cycles as a valid signal, and the information in the 8 cycle period will be lost. The deviation of DPSD calculation is caused by the non-synchronization in timing of the transmission and reception. Although shortening the amount of sample can compress calculating timing consume to some extent and reduce the probability of synchronization 
error generation, it will also detriment the accuracy of DPSD algorithm and increment the receiver system noise.

So the problem cannot be solved merely by reducing the sampling points. And PED system is actually applied in well down-hole remote unidirectional signal transmission mode. Since the received signal is full cycle sine wave, there is no rising edge triggered, which increases the difficulty in decoding messages.

When the transmitted signal is detected in the sampling window of time, it will cause significant changes in DPSD output amplitude values. Therefore, if the reception system captures 3 cycle of standard signal, the calculation time will be reduced to one receiver cycle. Above process supervise what is considered is the starting signal, and then Micro-controller shifts to high stability acquisition calculated reception mode with 25 cycles plus 8 . In consequence setting a code segment window interval to 33 cycles is reasonable, and the transmitting Timing Diagram figure is as follow.

Aiming at avoiding the situation of $0 / 1$ aliasing, the 8 cycles' time for recognizing blind zone is divided into two for the coding zone respectively. Then the code segment is sent correspondingly to increase the number of cycles of signal transmission time. As a result, the system demand for synchronization between the transmitter and receiver decreases, the error would only be made when there is more than 4 cycles' time from transmitter to receiver. So we can also ensure the accuracy of receiving information in mine by making start code, end code and checking code, all of which contribute to identifying code. At the same time, the comparison coding chart is supposed to be designed to make it convenient for encoded identification of daily information. As a result, underground production can be more efficiently by receiving the guidance from inoue and rescue for emergency will be in time.

Table 1 encoding table for daily information

\begin{tabular}{|c|c|c|c|c|}
\hline start bit & event type & operation & check code & stop code \\
\hline \multirow{2}{*}{ disaster precaution } & $\begin{array}{l}\text { gas explosion } \\
\text { Mine collapse }\end{array}$ & Channel one & \multirow{4}{*}{$\begin{array}{l}\text { Odd Parity } \\
\text { Check }\end{array}$} & \multirow{4}{*}{1111} \\
\hline & $\begin{array}{l}\text { water Penetration } \\
\text { Mine fire }\end{array}$ & Channel two & & \\
\hline \multirow{2}{*}{$\begin{array}{l}\text { produce } \\
\text { demanding }\end{array}$} & $\begin{array}{c}\text { aggregation } \\
\text { Lamp damage }\end{array}$ & Platform one & & \\
\hline & $\begin{array}{c}\text { Coal transport } \\
\text { equipment replacement }\end{array}$ & Platform two & & \\
\hline
\end{tabular}

After the test of system simulation experiment, the conclusion can be drew out that the system is of strong anti-jamming capability and the bit error rate is low. The speed of wireless signal transmission is $1.56 \mathrm{~s} /$ byte. Nevertheless, the existing system of PED synchronous the transmitter and receiver depending on precision clock signal and the system receives information by refreshing once per minute. The coding method proposed can ensure the system receive information timely which can reduce the cost and consumption of receiver to some extent.

\section{Summary}

The article illustrates each modular of wireless emergency communication system PED. Not only the hardware designed independently but also the phase-sensitive detection algorithm can further filter out low frequency interference. In addition, the creative timely coding design makes up the shortcoming where the error of identifying code is made when transmitting and receiving are not synchronous. In view of the simulation experiment of PED, the sensitivity is up to $0.75 \mathrm{~V} / \mathrm{nT}$ and attenuation of $50 \mathrm{~Hz}$ frequency interference reaches $45 \mathrm{db}$. The system can realize various function in information communication inoue underground in spite of the harsh environment where the SNR is up to $-58 \mathrm{db}$. 


\section{Acknowledgements}

This research was supported by National undergraduates innovating experimentation project (20 14A65301)

\section{References}

[1] PENG Li-jun. the development of PED underground wireless communication [J]. Sci-Tech Information Development \& Economy, 2003,05:168-169.

[2] Yl Bin LlU Yong CHEN Jing. Op Design of Through the-earth Mine Communication Receiving Circuit [J]. Ship Electronic Engineering, 2014,03,019.

[3] Shao Yingqiu, Cheng Defu, Wang Yanzhang, Zhang Fei. Research of high sensitivity inductive magnetic sensor [J]. Chinese Journal of Scientific Instrument, 2012,02

[4] GUO Wen-bin. Research on Through Rock Wireless Communication System for Emergency [D]. Harbin Institute of Technology 2012

[5] WANG Xiao-jun, ZHOU Xing-peng, WANG Yi. Research and Realization of DPSD Technique in Precision Impedance Analyzer [J]. Chinese Journal of Scientific Instrument, 2006,06:592-595

[6] Li ke, LU Bao-ping, ZHANG Jia-Tian. the Application and Research of Digital Phase-sensitive Detector in Logging Instrument [J], Petroleum Instrument, 2011.01;35-38+103.

[7] SU Ri-Jian, KONG Li, SHI Jun. Logging Instrument in the Design and Implementation of Digital Phase Sensitive Detection Algorithm [J]. Petroleum Instrument,,2009,11:60-62.

[8] SHI Jun, Cao Wei-Feng, XU Fang-you, LI Xiang-feng. The Realization of Digital Phase Sensitivity Detection Technique in DSP [J]. JOURNAL OF zhengzhou university of light industry(Natural Science), 2010,02:85-87.

[9] SHI Jun, Cao Wei-Feng, XU Fang-you, LI Xiang-feng. The Realization of Digital Phase Sensitivity Detection Technique in DSP [J]. JOURNAL OF zhengzhou university of light industry(Natural Science), 2010,02:85-87.

[10]LI Nian-qiang, Zhang Huan-chun, Yu Ai-qin. the Design and Implementation of A Digital Phase-sensitive Detector [J]. Electrical measurement \& instrumentation, 2001,05:29-30+7.

[11]LIU Yue, LIU Fu, DAI Yi-song. THE Research OF Reference signal frequency self-tuning digital phase-sensitive detector algorithm [J]. Journal of measurement,1998,04:74-78.

[12]ZHAO Zhan. The Design and Implementation of Underground Wireless Communication System[J]. Xidian University 2012.

[13]HUANG Jia-Liang. the Realization of Digital Phase-sensitive Detector Based on DSP [J], Petroleum Instrument, 2009,06:84-85. 